\title{
Germanica
}

\section{« La fuite sans fin » de Walter Mehring}

Walter Mehrings «Flucht ohne Ende»

\section{Pierre Giraud}

\section{OpenEdition}

Journals

Édition électronique

URL : http://journals.openedition.org/germanica/2807

DOI : 10.4000/germanica.2807

ISSN : 2107-0784

\section{Éditeur}

Université de Lille

\section{Édition imprimée}

Date de publication : 30 juin 1987

Pagination : 75-90

ISSN : 0984-2632

\section{Référence électronique}

Pierre Giraud, « «La fuite sans fin » de Walter Mehring », Germanica [En ligne], 1 | 1987, mis en ligne le 28 septembre 2018, consulté le 06 octobre 2020. URL : http://journals.openedition.org/germanica/ 2807 ; DOI : https://doi.org/10.4000/germanica.2807

Ce document a été généré automatiquement le 6 octobre 2020.

(c) Tous droits réservés 


\title{
« La fuite sans fin » de Walter Mehring
}

\author{
Walter Mehrings «Flucht ohne Ende»
}

\author{
Pierre Giraud
}

1 Ami de Kurt Tucholsky, juif lui aussi, comme lui collaborateur de la Weltbühne, la revue de Siegfried Jacobsohn, comme lui correspondant de presse à Paris (de 1925 à 1928 pour le Tagebuch de Leopold Schwarzschild), Walter Mehring doit quitter précipitamment l'Allemagne le 27 février 1933. Prévenu la veille par un fonctionnaire du Ministère des Affaires étrangères qu'un mandat d'arrêt allait être lancé contre lui, il a tout juste le temps de sauter dans le train à destination de Paris via Cologne où il apprendra l'incendie du Reichstag. Mehring est plongé brutalement dans la réalité de l'exil. Ainsi commence pour lui une période mouvementée qui deviendra toujours plus aléatoire à l'approche de la seconde guerre mondiale. Paris (1933-1934), Vienne (1934-1938), Paris encore, les camps d'internement de la Troisième République finissante et du gouvernement de Vichy, Marseille (1938-1941), les États-Unis enfin où il séjournera de 1941 à 1953, marqueront les différentes stations de ce qu'il faut bien appeler un calvaire dont il ne se remettra jamais. Pour Walter Mehring, la chute du Troisième Reich ne met pas un terme à l'exil. En témoigne la transposition lyrique de cette longue suite d'épreuves dans cinq recueils regroupés par l'éditeur Claassen sous le titre parfaitement adéquat emprunté d'ailleurs au poète lui-même : «Staatenlos im Nirgendwo $»^{1}$. Elle revêt des traits spécifiques qui distinguent très nettement Walter Mehring des autres exilés de la même génération, par exemple d'un Brecht auquel l'impossibilité du retour et la désespérance historique ou personnelle resteront toujours étrangères. Ces poèmes transmettent l'image d'une victime condamnée à jamais à l'errance, au déracinement, à l'absence de toute perspective et de tout salut.

2 Cette impasse n'apparaît pas d'emblée. La soudaineté des événements et le caractère absolu du national-socialisme libèrent d'abord chez lui comme chez Brecht une énergie nouvelle et une volonté résolue de combat. Dans l'exergue rimé qui ouvre l'ouvrage intitulé «Wir müssen weiler. Fragmente aus dem Exil» $»^{2}$, Mehring précise que son inscription sur la seconde liste des intellectuels proscrits constitue à ses yeux l'honneur suprême et 
d'ailleurs unique que lui ait jamais décernée son ancienne patrie. Notons au passage que cette dernière expression (Ex-Vaterland) indique bien que Mehring, contrairement à Brecht ou à d'autres semble déjà avoir rompu sans douleur tout lien avec elle et paraît exclure toute idée de retour. Pour l'instant cependant, l'heure est à la lutte et à la satire, une satire qui se veut défi, mais dont les vertus sont aussi roboratives :

Dass diese Zeit uns wieder singen lehre

Die guten Lieder eines bösen Spotts

- Selbst wenn uns Herz und Sinn danach nicht wäre

Nur euch zum Trotz!

(Nur Euch zum Trotz...!) $)^{3}$

Dans un autre poème, "Emigrantenchoral»" devenu selon le témoignage de la compagne de Mehring, l'actrice viennoise Hertha Pauli, l'hymne national des exilés allemands de Paris, le poète met en garde ses compagnons d'infortune en les incitant à ne pas céder à la nostalgie du pays natal. Au contraire, sur le ton de la dérision et dans un langage plein de verdeur, il les invite à aborder une ère nouvelle. Ils doivent désormais adopter une autre vision de la patrie, une vision plus vaste élargie aux dimensions de la solidarité humaine la plus universelle :

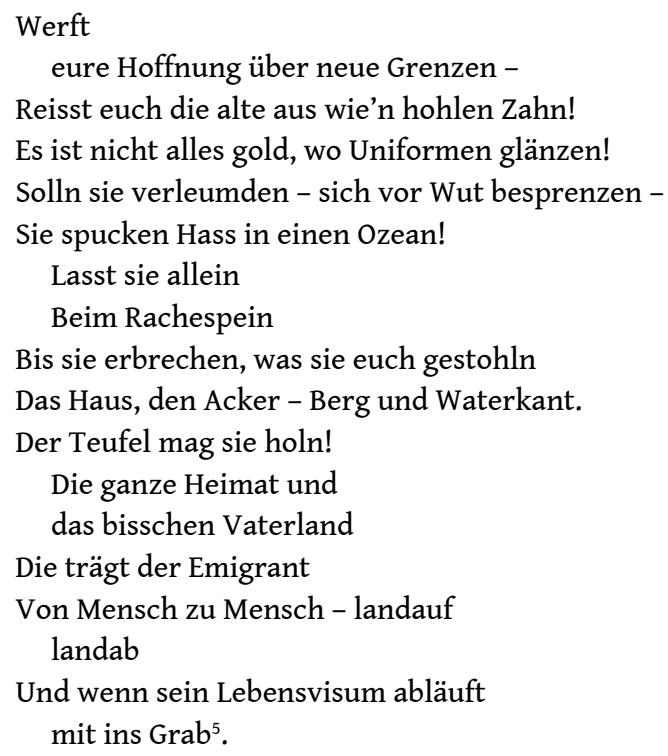

4 Combattre veut dire aussi dénoncer. La dénonciation sur le mode cocasse, grotesque ou parodique des slogans nazis, la mise en évidence de leur absurdité fournissent la substance essentielle des premiers poèmes. Ainsi dans «Burgfrieden $»^{6}$ où cette hydre qu'est l'art est sommée de garder son venin et d'épurer les écuries d'Augias de tous les pessimistes, cependant que le prolétaire n'a d'autre choix que de serrer la bride à son estomac pour l'empêcher de grogner et les Hébreux de rentrer leur nez pour éviter toute provocation et toute souffrance à ceux qui les environnent :

Denkt dran, ihr Kindlein, jung und alt,

dass weder Untat noch Gewalt

So provoziert wie

Leiden!

5 La révolution hitlérienne qui prétend susciter un homme nouveau annonce en fait une régression, un retour brutal au règne animal et à la sauvagerie de la jungle :

Der Mensch wird neu gestaltet

behelmt - gehörnt - behuft und gleichgeschaltet- 
GOTTgleichgeschaltet!

Die Dienstfreiwilligen marschieren

auf allen Vieren

Der Urwald ruft - 7

6 Comme l'atteste en particulier son livre de souvenirs, $L a$ Bibliothèque perdue ${ }^{8}$, Mehring a toujours été hanté par le monde fantastique des sorcières, des griffons, des licornes et des succubes. Il a dévoré toute une littérature marginale sur le bestiaire fantastique qu'il a étudié, nous dit-il, chez Aristote, les auteurs du Moyen Âge ou les disciples de madame Blavatsky. Il projetait d'écrire une zoologie des bêtes fabuleuses, de la mystique et de la superstition avant de s'interroger sur la survivance de ces monstres dans l'imaginaire humain, la littérature et tout particulièrement l'arsenal de métaphores chères au journalisme politique. Hanté au double sens du terme, c'est-àdire à la fois tenté par les arrière-plans que cet univers ouvre à la littérature et à la psychologie et effrayé par ses incarnations sur la scène politique.

7 On ne s'étonnera donc point de le voir présenter le nazisme comme l'irruption des monstres dans un monde organisé tant bien que mal par la raison, comme le triomphe du non-esprit (Ungeist) sur l'esprit (Geist) et la culture. Ainsi le vieil épouvantail pour les oiseaux, qui est dépourvu de tête, de peau et de corps, se dresse dans le paysage comme un inoffensif fantôme de chiffons, mais voilà soudain qu'une vie nouvelle lui est insufflée et que sous l'effet des miasmes surgis de la nuit peuplée de fantômes, il enfle démesurément, devient la "terreur des nations » (Völkerschreck) et un épouvantail pour les hommes (Menschenscheuche) ${ }^{9}$. Un autre poème ${ }^{10}$ retrace sous la forme d'un conte la genèse de ce que Brecht appellera la bête immonde, chez Mehring un basilique venimeux, endormi sur une hache de bourreau, qui, soudain, se met lui aussi à enfler :

Er wuchs und schwoll und krähte geil;

Horcht! Horcht! Das ist sein Schrei... heil heil ...

(Der Basilisk Heilheil!)

Plusieurs poèmes sont construits selon une structure analogue qui décrit la même évolution de l'infiniment petit à l'infiniment grand, du chétif au monstrueux: le batracien qui veut se faire aussi gros que l'auroch. Qualifié de loupé sur le plan de la race par le marais où il a été conçu, il décide de s'exercer au pas de l'oie, à la flexion des genoux et à la technique de la vague d'assaut avant d'éclater comme une baudruche, ce qui constitue une des rares notes optimistes du recueil ${ }^{11}$.

9 Bientôt toute l'Allemagne ne sera plus qu'un zoo aryen ${ }^{12}$ d'ailleurs pire que la jungle puisque certaines espèces doivent en être immédiatement et définitivement éliminées. Les animaux allemands, vers et vermines, doivent bannir de leur fourrure tout élément allogène et ne plus tolérer en particulier que le juif se vautre dans la fange allemande. Il faut libérer le troisième "Tierreich» de toute souillure étrangère. Ce programme d'épuration ne suffit d'ailleurs pas, car certaines espèces, fussent-elles aryennes, sont déjà contaminées et menacées de dégénerescence comme, par exemple, les sauriens de Wotan ou les dragons d'Odin qui de la gueule au croupion sont dépourvus de cervelle et dont la descendance fournit le gros de la racaille pacifiste. Il est dont tout à fait urgent de lancer un vaste programme de régénération :

Züchtet Übermenschen-Affen

Führer-Mammuts, kolossal

Wie sie Gatt im Zorn erschaffen

Aus dem Opernarsenal ${ }^{13}$. 
Comme Brecht, Mehring parodie les chants et les slogans nazis ou les cantiques protestants pour souligner que l'arrivée de Hitler au pouvoir instaure le règne de la violence nue. La création de celui qui se veut l'égal de Dieu et qui n'est jamais nommément cité, sinon par périphrase, est une œuvre de destruction, un viol délibéré de toutes les valeurs, un saccage absolu :

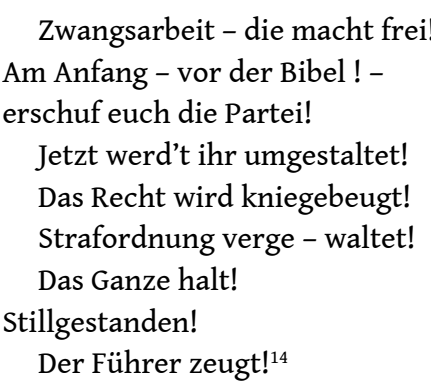

Déjà la guerre règne en temps de paix ${ }^{15}$. Le monde est inversé. Incontestablement, cette verve parodique et ces sarcasmes sont portés par un élan destructeur et libérateur. Réduire l'adversaire par la dérision systématique à la seule dimension qui lui convienne est une manière d'indiquer que le combat garde un sens. Ces vers de Mehring perpétuent le mouvement dit du «lyrisme fonctionnel» (Gebrauchslyrik) qu'avec Kästner, Tucholsky et Brecht (pour s'en tenir aux noms les plus célèbres) il avait brillamment illustré durant la République de Weimar. Il s'agissait alors pour eux de montrer que le lyrisme ne pouvait être séparé de sa fonction politique, de son pouvoir opérationnel sur les esprits. Il devient une arme destinée à provoquer chez le lecteur ou l'auditeur un sursaut de réflexion, un éveil de la conscience à l'actualité et à la gravité des problèmes de l'heure. Autant que par son contenu, un tel lyrisme se définit par son rapport au public. Or l'exil modifie radicalement cette relation. Coupé brutalement de son audience potentielle, le poète animé d'une telle ambition, ne peut qu'éprouver rapidement la vanité de son entreprise. Tous les poètes allemands exilés de l'époque en ont fait l'amère expérience plus ou moins rapidement. Nul ne l'a faite aussi tôt (dès 1934) et avec une telle acuité que Walter Mehring.

Outre l'espoir de voir la situation se modifier à terme, la satire délibérée et plus ou moins jubilatoire implique une certaine distance par rapport à la réalité dénoncée. Elle est le fait d'un spectateur qui, certes, n'est pas détaché puisqu'il veut agir par le verbe, mais qui, du fait même de l'exil, doit échapper à toute forme de persécution ou de suspicion. Or, très vite, et son lyrisme en fait foi, Mehring découvre que l'exilé, s'il est provisoirement rescapé, bute à l'étranger, dans son pays d'accueil (en l'occurrence la France) sur une réalité qui fait de lui d'une manière feutrée et insidieuse une victime, un indésirable, un paria.

Dans la suite de poèmes que Walter Mehring écrit entre 1933 et la fin de la guerre et qu'il publie dans différentes revues allemandes de l'exil ${ }^{16}$, on ne peut distinguer plusieurs phases: une phase de combat et une phase de dépression. Très tôt les deux tonalités s'entremêlent, s'entrecroisent, coexistent. Certes, comme Brecht, Walter Mehring, tout en dénonçant vigoureusement le Troisième Reich, se fait aussi le chroniqueur des événements essentiellement tragiques de l'actualité ou annonciateurs de futures catastrophes: Guernica ${ }^{17}$, l'assassinat d'Erich Mühsam ${ }^{18}$, les menaces qui pèsent sur la vie de Carl von Ossietzky ${ }^{19}$, le rapprochement anglo-allemand ${ }^{20}$ ou germano-soviétique ${ }^{21}$ ou enfin le long martyrologue de ses amis écrivains assassinés ou suicidés $^{22}$. Il n'en reste pas moins que, dès 1934, la dure réalité quotidienne de l'exil 
tient une place importante qui ne fera ensuite que s'accentuer. Il est bien loin l'espoir que Mehring invitait ses compagnons d'infortune à projeter vers de nouvelles frontières dans l'Emigrantenchoral.

À peine arrivé dans ce qu'il croit être un pays d'accueil digne de confiance et d'admiration, l'exilé fait rapidement l'amère constatation qu'il est d'abord considéré comme un suspect prié plus ou moins brutalement de présenter ses papiers ou d'en obtenir de nouveaux :

Ich grüsse Dich Fremde - sei Heimat mir!

- Zeigt Euren Pass - zeigt Euer Papier!

Man nahm mir Pass Weib Kind und Gut

Seht mich doch an! Hier rinnt mein Blut...

Wir sehen es - doch nur mit Papiern

Kann Euer Blut die Grenze passiern! ${ }^{23}$

Le voilà désormais astreint à une quête incessante et humiliante auprès d'une bureaucratie toute puissante pour qui les êtres humains se réduisent à des dossiers et à des matricules. Il a progressivement conscience de subir un processus de dépersonnalisation et même de désincarnation (Und sieh und siehe! Die Akten schwolln / Er wandelte lebhaft in Protokolln) ${ }^{24}$. Bientôt, on le somme de quitter sous la menace d'une amende "son tombeau d'accueil avec ses ossements». Si le nazisme selon Mehring, peut être décrit comme la résurrection des monstres, la réalité existentielle de l'exilé se confond avec l'inconsistance des spectres. La négativité est son lot (Die Wache gab ihm einen Stoss. / Da stand der Mann im Staatenlos) ${ }^{25}$. Loin de trouver une nouvelle patrie, il est privé d'identité, rejeté dans le flou de l'inexistence et la précarité.

Une précarité qui est d'abord matérielle. Si Brecht, dans ses poèmes d'exil, évoque, même brièvement, en quelques touches concrètes son cadre de vie, singularise par l'évocation d'un arbre ou d'un paysage la maison amie où il a trouvé refuge, mentionne les quelques menus objets d'usage quotidien qui lui appartiennent en propre et qui le suivent dans toutes ses pérégrinations par delà les frontières, on cherchera en vain des notations comparables chez Walter Mehring. Son univers est celui des petits hôtels minables et anonymes qu'il décrit dans un poème de $1934^{26}$ et auxquels il se sait désormais condamné à perpétuité. Délabrées, hantées par les fantômes des hôtes de passage et des gnomes inquiétants, leurs chambres sont recouvertes de tapisseries d'où semblent émerger des tentacules prêts à étouffer le nouvel occupant qui donc a le sentiment de camper dans l'antichambre de la mort. La terre d'asile est la préfiguration du tombeau.

Pour l'écrivain, la précarité matérielle se double rapidement d'un autre constat douloureux: l'incompréhension. Alors qu'il se raccroche à l'écriture comme à sa dernière planche de salut (Noch fasste ich, ans Wort gekrallt, / Im Schreiben meinen letzten Halt.... ${ }^{27}$ et qu'il se sent investi de la mission de lancer un cri d'alarme face aux dangers qui depuis l'Allemagne hitlérienne, menacent les autres pays européens et en particulier la France, il se heurte à l'incrédulité et à l'indifférence. Comme Cassandre, les exilés parlent dans le vide et ne peuvent faire passer leur message (Wir ausgesandt als Warnungszeichen / Hörn ungenützt die Zeit verstreichen) ${ }^{28}$. Ils ressemblent à la statue de ce saint dont Mehring retrace dans un poème de $1936^{29}$ la légende et le martyre: Sankt Heimatlos. Dressé sur la frontière, privé de ses mains qu'il a usées à écrire, il n'a pas la moindre offrande à présenter :

Da hält er ohne Hände

Der Sprache Dornenkranz 


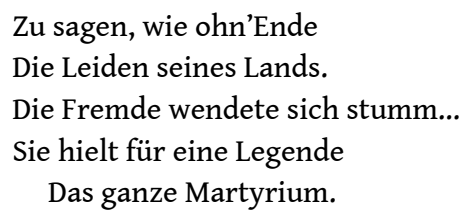

Des épreuves encore plus redoutables attendent bientôt l'exilé et ses semblables. Après la déclaration de la guerre en septembre 1939, les gouvernements français, celui de la Troisième République et le gouvernement de Vichy, croient bon, pour des raisons de sécurité, de regrouper dans des camps d'internement environ 300 émigrants dont Walter Mehring qui est incarcéré au camp de Falaise en Normandie. Libéré en février 1940, il sera à nouveau arrêté en septembre 1940 après une tentative manquée de passage en Espagne. Il est alors détenu au camp de Saint-Cyprien d'où parvient encore à le tirer au bout de deux mois grâce à ses relations diplomatiques et à l'intervention d'un avocat français le président de l'«Emergency Rescue Committee» à Marseille, Varian Fry (c'est également grâce à lui que Chagall pourra gagner les États-Unis). Toujours grâce à Fry qui le cache désormais dans son bureau, Mehring parviendra à quitter illégalement Marseille le 3 février 1941 à bord du paquebot "Wyoming " et débarquera le 26 février 1941 à Miami après une courte escale à la Martinique. Cette période, relativement brève dans le temps mais interminable pour celui qui la subit, fait l'objet des « 12 lettres de minuit $»^{30}$.

19 À l'exception des trois premières écrites à Vienne et qui prennent la forme de cauchemars prémonitoires, ces poèmes traduisent le désarroi absolu de l'exilé qui se sait désormais doublement traqué puisque la France qu'il a tant aimée et sur laquelle il a tant misé, le trahit et se trahit en "déclarant la guerre aux victimes" Alternativement voué à l'internement et à la clandestinité, il se sent soustrait à la sphère de l'existence, plus exactement placé dans une zone indécise entre la vie et la mort. Ainsi au camp de Falaise où s'abolissent les notions de temps et d'espace (Nicht Tag, nicht Nacht... und kein Wohin) ${ }^{32}$. Par comparaison, la promiscuité des "petits hôtels » peut paraître rétrospectivement supportable. Cernés par une végétation de barbelés (la végétation du vingtième siècle, dira Mehring dans les Fragmente aus dem Exil, p. 91), entassés comme des animaux sur des litières de paille pourrie rongée par la vermine, guettés par la folie, écartelés par les interrogatoires inquisiteurs d'une "police infernale ", disséqués et transformés en dossiers, ils ont le sentiment que leur vie n'avance plus, fait du sur place dans le labyrinthe d'un minotaure à tête de colonel de l'armée française qui, le soir venu, retourne à son hôtel pour sacrifier aux petits plaisirs de la vie.

L'enfermement lui est d'autant plus insupportable qu'il se double d'un drame personnel : la séparation brutale de l'être aimé (Hertha Pauli) auquel il sait ne pouvoir renoncer et qui, avec l'écriture, restait son seul lien fort avec la vie. Séparation d'autant plus poignante que le poète feint de la présenter comme la simple conséquence de son incarcération, alors qu'il s'agit en fait d'une rupture décidée par sa partenaire :

Dass mich zuletzt noch ins Exil

Die Liebe stiess: das war zu viel!

Das ging zu weit! Noch der Verzicht

Auf Dich! Nein! Ich ertrag es nicht.

So nimm, Nacht, den schon lebensleeren

Leib, um mich völlig zu entschweren ${ }^{33}$. 
lettres de minuit dédiées à Hertha Pauli et qualifiées par Hermann Kesten de " poèmes d'amour apocalyptiques $»^{34}$ mêlent ces deux sources de dépossession : drame politique et drame personnel. Elles déroulent un cauchemar devenu réalité et nous entraînent à la suite du poète dans une plongée inexorable, une descente au royaume des ombres: Träum ich? Nein! Wahr ist, was mir schien / Ausgeburt kranker Phantasien ${ }^{35}$.

La végétation de barbelés qui le cerne de toutes parts, les masques lémuriens des codétenus dont la denture est rongée par le scorbut, ces résidus humains poussés à coups de gourdin au travail forcé par la grande mobile et qui se battent comme des bêtes pour une pitance souillée sont autant de signes indubitables qui lui rappelle qu'il est soustrait au monde humain et que la notion de l'humain a déserté le monde :

Jawohl! Das Unten kommandiert.

Nach unten wird die Welt regiert.

Am Grund des Alls hat sein Revier

Der Zwischengott - das Übertier ${ }^{36}$.

Les exilés sont coupés de la terre des hommes, elle-même déchue et de toute façon hostile. Libérés provisoirement, ils ne retrouvent pas pour autant leur statut d'homme, mais sont guettés par mille dangers, voués à la suspicion, plongés dans un univers glauque où la garde mobile lance ses filets pour pêcher l'étranger ${ }^{37}$. Même les éléments naturels ont partie liée contre eux et pactisent avec les nouveaux démons. Dans le Marseille nocturne où se retrouvent les fêtards autour d'huîtres et d'oursins, la mer, tapie dans l'ombre, s'apprête à engloutir les bannis qui croient avoir trouvé leur salut sur un bateau clandestin. Le vent du Nord-Ouest bouscule sans ménagement l'homme traqué qui s'approche de la jetée et déverse sur sa tête une pluie aussi noire que l'encre des bureaucrates :

Nord-West, der knotige Tyrann,

Pöbelt mich längs dem Hafen an,

Giesst über mich in windigem Hass

Die Schwärze aus dem Tintenfass ${ }^{38}$.

Refoulées sur le "rivage des touristes sans destination" (Ausweglos-Touristen), ces ombres ont encore la volonté d'échapper à leur bourreau et sont encore animées par la volonté de fuir, mais une telle tentative ne débouche sur aucune perspective et se voit condamnée d'avance à l'échec :

Es geht ein Schiff um Mitternacht.

Ertrunkene haben's klar gemacht.

Legt nirgends an.Klock Nimmermehr.

Sein Kapitän heisst Ahasver ${ }^{39}$.

Plus généralement, l'idée même du retour a perdu toute espèce de sens. Elle n'a plus de raison d'être. Elle aussi est bannie et chassée à jamais de l'Europe devenue prison et aussi du monde méditerranéen où l'esprit de l'Antiquité est porté disparu, victime d'une armée de pygmées qui ont jeté bas l'olympe et chassé Apollon, où les Phocéens sont devenus des " cannibales administratifs ", où Ithaque s'est évanouie à jamais :

Unerlaubt hier - und fort nicht darf...

Um Pässe fleht nach Übersee

Auf einer Bettel-Odyssee...

Und keiner Heimkehr Hoffnungsfunken

- Sein Ithaka ist längst versunken.... ${ }^{40}$. 

Mehring parviendra finalement à gagner les États-Unis où il restera douze ans (jusqu'en février 1953) confronté à de graves difficultés matérielles que son ami Georg Grosz l'aide à surmonter aux moments les plus critiques. Jamais il ne réussira à trouver dans ce pays une seconde patrie comme l'indiquent les poèmes peu nombreux composés à cette époque et regroupés en 1951 sous le titre parfaitement explicite : Arche Noah S.O.S. Outre la pauvreté, l'exilé a immédiatement le sentiment de buter sur les mêmes obstacles qui restent d'autant plus infranchissables qu'ils sont plus insidieux. Il continue d'éprouver une affinité instinctive pour la figure du pauvre voyageur balloté par le destin (der Wanderer) qui, comme celle du marin, traverse toute son œuvre lyrique. Il en a croisé des milliers d'exemplaires. Une voix impérieuse et cachée leur commande de poursuivre leur route sans se retourner dans une fuite éperdue à laquelle la «meute aux yeux de loup $»^{42}$ viendra tôt ou tard mettre un terme :

So verurteilt, wandernd den Lebensweg abzubüssen,

Vorwärts - vorwärts in immer verlangsamter Hast

Mit schon beerdigten Füssen

Tiefer und tiefer ${ }^{43}$.

Alors qu'il devrait se réjouir d'être sauvé et cesser de récriminer contre les désagréments ou les tares de sa terre d'accueil ${ }^{44}$, son malaise persiste et il continue de se sentir autre. Le bac délabré qu'il observe depuis la rive de l'Hudson et qui se veut symbole de la destinée du poète dérive dans le brouillard vers des horizons flous et indéfinis avant de s'évanouir bientôt dans le vide pour ne plus jamais revenir :

Wohin schreibst du...?

Schau hin! Gerad verschwand die Fähre

Im Nebelfaltenwurf - mein Wort an Bord ...ins Leere... ${ }^{45}$.

S'il n'est pas happé par les tourbillons de la dérive, il a le sentiment d'être pétrifié, immobilisé, magnétisé comme l'indique le titre anglais (Spellbound) du poème qui établir un parallélisme frappant entre la destinée des Peaux Rouges expulsés de leurs terres par des blancs eux-mêmes émigrants et sa propre destinée : une fuite perpétuelle sans autre perspective que celle de la mort:

Nun betteln wir als Letzte um Asyl

Bei Ausgewanderten: wir Europäer,

Erblich belastet - welcher Zukunft Ahnen?

Wohin, Nachfahren, wandern eure Karawanen? ${ }^{46}$

Les démons imprévisibles restent les plus forts et l'homme, leur esclave, ne pourra jamais leur échapper ou les vaincre :

Erschöpft, absaufend in der Todesbucht -

gegen den Strom anschwimmend - auf der Flucht

Vor sich - vor seiner Liebe - Seines gleichen ${ }^{47}$,

31 Condamnée à l'errance perpétuelle dans le dénuement et la solitude, ces bannis empruntent des routes qui toutes mènent à la mort :

Die Wege vereisen

Die Wagen entgleisen

Und alle Reisen enden

Im Rauhwald aus Legenden 
Wo sie an Warnungsschildern:

«Dead End», «End-Weg»

verwildern $^{48}$.

Europe et qui sont regroupés sous le titre «Letzte Gedichte aus dem Ketzerbrevier» ne font que confirmer cet accablement et ce pessimisme. On y retrouve la récurrence des mêmes thèmes et des mêmes métaphores : l'exode, les cauchemars devenus réalité, l'arche à la dérive, la solitude irrémédiable, des prières désespérées comme cette treizième et dernière lettre de minuit en forme d'épitaphe placée sous le signe de François Villon que Mehring a toujours considéré comme un frère :

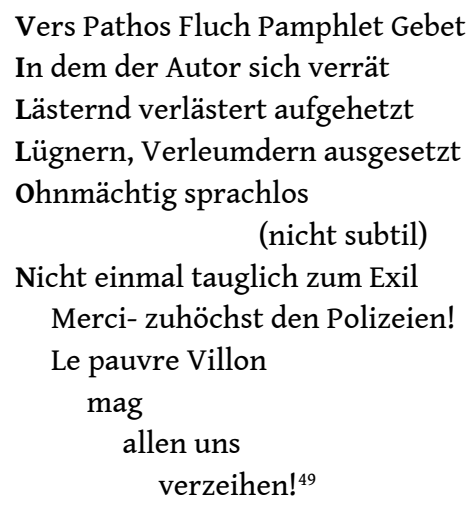

Dès les premiers recueils de la période weimarienne, le thème de l'errance ou du vagabondage concrétisé par la figure du matelot et traité sur le mode enjoué ou doux amer a toujours occupé une grande place dans le lyrisme de Walter Mehring. Mais à partir de 1933, il s'agit de bien autre chose. Non seulement le thème est dominant et même omniprésent, il sert également de vecteur à une vision désespérée de l'existence. Non seulement, Walter Mehring est devenu « inapte à l'exil », comme il le dit dans le dernier poème que nous venons de citer, mais aussi inapte à retrouver des racines, un ancrage, une identité.

Avec plus ou moins de bonheur, on a tenté de dresser une typologie des œuvres lyriques écrites par les exilés. Pour Walter Mehring, une telle tentative est loin d'emporter la conviction. Son cas est irréductible à tout autre et se différencie très nettement de celui d'un Brecht. Pour ce dernier, l'exil, aussi pénible et aussi inconfortable soit-il, n'est finalement qu'une parenthèse avant l'effondrement - jugé inéluctable - du Troisième Reich. Pour lui le dernier mot n'est pas dit. L'heure du retour sonnera un jour, un retour douloureux certes, puisque le fils prodigue sait qu'il reviendra dans le sillage destructeur des avions alliés, mais ouvert à l'espoir et aux promesses de reconstruction ${ }^{50}$.

On ne trouve rien de tel chez Walter Mehring. Pour lui, l'exil reste une expérience irrémédiable, un traumatisme insurmontable, une inexorable mort lente imposée. L'exil est synonyme d'enfermement. L'exil a brisé son rapport à la réalité et lui interdit toute issue vers l'utopie d'un monde meilleur. La confession désespérée de cet homme indique enfin qu'il est beaucoup plus que le poète de cabaret auquel on a voulu le réduire. S'il n'a pas suivi dans le suicide ses amis Tucholsky et Toiler, la fuite sans fin qui fut son lot est tout aussi poignante, particulièrement pour un lecteur français. 


\section{NOTES}

1. Walter Mehring, Staatenlos im Nirgendwo, Die Gedichte, Lieder und Chansons 1933-1974, Claassen Vertag, Düsseldorf, 1981. Hrsg. von Christoph Buchwald. Abréviation: W.M.S.N.

2. Walter Mehring, Wir müssen weiter. Fragmente aus dem Exil. Claassen Verlag, Düsseldorf, 1979, Hrsg. von Christoph Buchwald.

3. Und Euch zum trotz. Aus: W.M.S.N., p. 15.

4. Idem, Anmerkung 17, p. 246.

5. Ibid., Der Emigrantenchoral, pp. 18-19.

6. Ibid., Burgfrieden, pp. 20-21.

7. Ibid., Lied der Arbeitsdienstler, pp. 21-22.

8. Walter Mehring, La Bibliothèque perdue, Julliard, Paris, 1958, traduit par Gilberte Marchegay, préface de Robert Minder.

9. W.M.S.N., Die alte Vogelscheuche, pp. 36-38.

10. Ibid., Der Basilisk. Ein Wintermärchen, pp. 57-59.

11. Ibid., Von der Kröte, die gross wie ein Ochs sein wollte, pp. 50-51. Cf. aussi : Der Überknirps, pp. 38-39.

12. Ibid., Ader-Zoo, pp. 33-34.

13. Ibid., Ader-Zoo, p. 34.

14. Ibid., Lied der Arbeitsdienstler, p. 22.

15. Ibid., Krieg im Frieden, pp. 23-24.

16. On en trouvera la liste dans les notes de l'édition Claassen (citée plus haut) établies par Christoph Buchwald.

17. W.M.S.N., Hymne auf den Sieger von Guernica, pp. 78-80.

18. Idem, Erich Mühsam, p. 54.

19. Ibid., Nächtlicher Rat für C.v.O., pp. 69-70.

20. Ibid., Ein Friedensnachttraum, pp. 76-77.

21. Finnland, p. 87.

22. Ibid., X. Brief aus der Mitternacht (Marseille, Silvester 1940/1941, in memoriam), pp. 129-132.

23. Ibid., Mirakel des Heiligen Bürokratius, p. 29.

24. Ibid., Mirakel des Heiligen Bürokratius, p. 30.

25. Ibid., Mirakel des Heiligen Bürokratius, p. 29.

26. Ibid., Die kleinen Hotels, pp. 40-41.

27. Ibid., Briefe aus der Mitternacht, IV, Nice, juni 1939, p. 112.

28. Ibid., Briefe aus der Mitternacht, V, Camp de Falaise. Herbst/Winter 1939, p. 117.

29. Ibid., Standbild an einer Grenze, pp. 66-67.

30. Ibid., 12 Briefe aus der Mitternacht, pp.99-136. Publiées pour la première fois en édition bilingue (anglais-allemand) avec des dessins de Georg Grosz chez l'éditeur new-yorkais Curl Inc. en 1944 sous le titre No road back - Kein Weg zurück.

31. Ibid., VII, Brief, p. 123.

32. Ibid., V, Brief, p. 115.

33. Ibid., VI, Brief, p. 121.

34. Ibid., cité d'après Christoph Buchwald, p. 258. W.M.S.N.

35. Ibid., VII, Brief, p. 123.

36. Ibid., VIII, Brief, p. 125.

37. Ibid., p. 125.

38. Ibid., p. 125.

39. Ibid., IX, Brief, p. 127. 
40. Ibid., VIII, Brief, p. 127.

41. Ibid., XI, Brief, p. 133.

42. Ibid., p. 134.

43. Ibid., Der Wanderer, p. 151.

44. Ibid., Rescue-Visa, pp. 152-153.

45. Ibid., Riverside-Drive, pp. 153-156.

46. Ibid., Spellbound, p. 169.

47. Ibid., Spellbound, p. 169.

48. Ibid., Wanderung for ever, p. 168.

49. Ibid., Epilog: Dreizehnter und letzter Brief aus der Mitternacht, p. 212.

50. Voir en particulier les poèmes: Die Hoffnung der Welt, pp. 738-739, Über Deutschland, p. 752, Finnische Landschaft, p. 822, Die Rückkehr, p. 858. Cités d'après l'édition Die Gedichte von Bertolt Brecht in einem Band, Suhrkamp, I. Auflage, 1981.

\section{RÉSUMÉS}

Dès février 1933, l'exil ouvre pour Walter Mehring une longue série d'épreuves qu'il ne surmontra jamais comme l'indique l'analyse des cinq recueils lyriques regroupés sous le titre Staatenlos im Nirgendwo (Apatride dans l'État de nulle part). La transposition lyrique de ce calvaire revêt des traits spécifiques qui distinguent très nettement le poète des autres exilés de la même génération, en particulier de Brecht. Certes, les premiers poèmes de tonalité satirique, traduisent encore la volonté de combattre le Troisième Reich présenté comme le retour des démons et le règne de l'animalité, mais rapidement l'espoir cède la place à l'impuissance et au pessimisme absolu. Dans les différents pays « d'accueil », particulièrement en France, l'exilé est en butte à la sacro-sainte bureaucratie, mais aussi, très vite, à la persécution et à l'internement. Toute perspective de salut est exclue. Le retour à Ithaque, de toute façon impossible, a, en outre, perdu toute sorte de sens, puisque Ithaque elle-même a "sombré depuis longtemps ». Tout à la fois banni et «inapte à l'exil ». Le poète est condamné à une "fuite sans fin ", particulièrement poignante pour un lecteur français.

Bereits ab 1933 eröffnet das Exil für Walter Mehring eine lange Reihe von Prüfungen, die er niemals überwinden wird, wie dies aus der Analyse der fünf unter dern Titel Staatenlos im Nirgendo zusammengestellten Gedichtsammlungen hervorgeht. Die lyrische Übertragung dieses Leidensweges setzt neue spezifische Akzente, die den Dichter von den anderen Exilierten der gleichen Generation, insbesondere von Brecht, sehr deutlich unterscheiden. Zwar spiegeln die ersten Gedichte satirischer Tonart immer noch den Willen wider, das Dritte Reich, das als Rückkehr der Dämonen und als Rückfall in das Tierische dargestellt wird, zu bekämpfen, aber die Hoffnung weicht sehr schnell der Machtlosigkeit und dem absoluten Pessimismus. In den verschiedenen «Aufnahmeländern», besonders in Frankreich, ist der Exilierte zuerst dem sakrosankten Bürokratismus, dann sehr schnell der Verfolgung und der Internierung ausgesetzt. Jede Aussicht auf Erlösung bleibt versperrt. Die ohnehin unmögliche Rückkehr nach Ithaka hat darüber hinaus jeden Sinn verloren, da Ithaka selbst «längst versunken» ist. Zugleich vertrieben und nicht einmal zum Exil tauglich, bleibt der Dichter auch nach 1945 einem unheilbaren Trauma verhaftet und ist $\mathrm{zu}$ einer «Flucht ohne Ende» verdammt, deren Lektüre besonders einen französischen Leser tief beeindruckt. 
AUTEUR

PIERRE GIRAUD

Université de Lille III 